Project Title: Can latent class analysis be used to improve the diagnostic process in pediatric patients with chronic ataxia?

Primary Supervisor Name and Department:

Michael S Salman, Department of Pediatrics and Child Health

Co-Supervisor Name and Department:

N/A

SUMMARY: (no more than $\mathbf{2 5 0}$ words single spaced)

Background: Ataxia, defined as unsteady stance or impaired coordination of voluntary movement, is classified according to its presentation as acute, sub-acute, or chronic. Chronic ataxia is a relatively common presentation in children. There are numerous causes of chronic ataxia, making it difficult to derive a diagnosis in a timely manner.

Objective: We hypothesized that the efficiency of the diagnostic process can be improved with systematic analysis of clinical features in pediatric patients with chronic ataxia.

Methods: A cohort of 184 patients, aged 0-16 years with chronic ataxia who received medical care at Winnipeg Children's Hospital during 1991-2008, was ascertained retrospectively from several hospital databases. Clinical details were extracted from hospital charts. The data were compared among the more common diseases using univariate analysis to identify pertinent clinical features that could potentially improve the efficiency of the diagnostic process. Latent class analysis was then conducted to detect unique patterns of clinical features, and to determine whether these patterns could be associated with chronic ataxia diagnoses.

Results: Two models each with three classes were chosen based on statistical criteria and clinical knowledge for best fit. Each class represented a specific pattern of presenting symptoms or other clinical features. The three classes corresponded to a plausible and shorter list of possible diagnoses. Conclusions: Specific patterns of presenting symptoms or other clinical features can potentially aid in the initial assessment and diagnosis of pediatric patients with chronic ataxia. This will likely improve the efficiency of the diagnostic process.

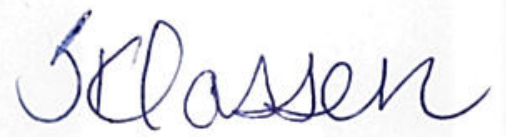

Student Signature

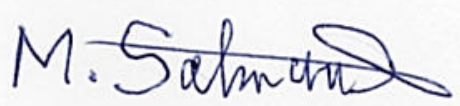

Supervisor Signature

ACKNOWLEDGEMENTS:

I gratefully acknowledge the support by one or more of the following sponsors;

CancerCare MB

H.T. Thorlakson Foundation

Dean, College of Medicine

Research Manitoba

Children's Hospital Research Institute of MB

Kidney Foundation of Manitoba
Manitoba Medical Service Foundation Associate Dean (Research), College of Medicine

Heart and Stroke Foundation

Health Sciences Centre Research

Foundation

Other:

June Helen Coulter Memorial Scholarship 
Samantha Klassen

\section{Can latent class analysis be used to improve the diagnostic process in pediatric patients with chronic ataxia?}

\section{Introduction and Background}

Ataxia is defined as unsteady stance or impaired coordination of voluntary movement. It is caused by disorders of the central nervous system, peripheral nervous system, or both. Cerebellar ataxia is caused by dysfunction of the cerebellum, or its input or output tracks. ${ }^{1}$ Sensory ataxia is caused by interruption of proprioceptive input to the brain, and is usually caused by disorders affecting the peripheral nerves, dorsal root ganglia or posterior columns of the spinal cord. ${ }^{1-3}$ Incoordination caused by decreased level of consciousness, extrapyramidal movement disorder, spasticity, muscle weakness, skeletal disease, or psychiatric disorder does not constitute ataxia.

Ataxia can be classified according to its mode of presentation as acute, sub-acute, or chronic ataxia. The latter can be further classified into non-progressive, progressive or recurrent ataxia. Chronic ataxia is a relatively common presentation in pediatrics with an annual crude incidence rate of 3.2 per 100,000 and annual crude period prevalence rate of 2.4 per 10,000 children less than 17 years of age in Manitoba. ${ }^{4}$

Ataxia may be associated with abnormal eye movements, impaired articulation of speech, swallowing difficulties, and poorly coordinated upper limb movements and gait. Children with chronic ataxia may have additional co-morbidities such as epilepsy, developmental delay, extrapyramidal movement disorders, feeding difficulties, and speech/ language delay. ${ }^{4}$ These co-morbidities can further adversely affect the development of the child and make activities of daily living more difficult. In addition, the mental and physical disabilities encountered by these children negatively impact the whole family and impose a significant financial burden on them and on the health care system. ${ }^{5}$

There are many disease processes that can cause ataxia including infectious, post infectious, demyelinating, autoimmune, vascular, metabolic, traumatic, toxic, cerebellar or cerebral malformations, neoplastic, paraneoplastic, and genetic (autosomal recessive, dominant, $X$-linked, and mitochondrial inheritance). ${ }^{1,2,6}$ The diverse etiologies and the numerous diseases associated with chronic ataxia make it difficult to derive a diagnosis early in the course of the disease, because ataxia may be the sole symptom, ${ }^{3}$ at least initially. In addition, there is considerable overlap of the clinical features among the various diseases that cause chronic ataxia, which complicates the clinical assessment. ${ }^{6}$ Therefore, the cost of assessing patients with chronic ataxia is considerable ${ }^{5}$ because of the need for multiple clinic follow up visits and extensive investigations.

There are several published reviews on the assessment of pediatric patients with ataxia based on case reports, small case series, personal experience, ${ }^{2,6,7,8}$ and neuroimaging findings in the posterior fossa such as cerebellar atrophy, ${ }^{9,10}$ or cerebellar hypoplasia. ${ }^{11}$ Many studies have concentrated solely on the assessment of inherited ataxia, ${ }^{3,12,13}$ or

\footnotetext{
Abbreviations:

AIC: Akaike Information Criteria

BIC: Bayesian Information Criteria

EEG: electroencephalogram

HIE: hypoxemic ischemic encephalopathy after birth

JS: Joubert syndrome and related disorders

LCA: latent class analysis

MRI: magnetic resonance imaging

NCL: neuronal ceroid lipofuscinosis

NMD: neuronal migration disorder

NYD: not yet diagnosed
} 
specific diseases with ataxia..$^{8,14}$ We did not find any studies that systematically investigated the clinical features of a large cohort of children with diverse causes of chronic ataxia. Furthermore, clinical studies aiming to improve the diagnostic process of pediatric patients with chronic ataxia are lacking. We hypothesized that the efficiency of the diagnostic process can be improved with systematic analysis of clinical features in a large cohort of pediatric patients with chronic ataxia. The objectives of our study were to: 1) Describe and quantify the clinical findings in children and adolescents with chronic ataxia according to their diagnoses, 2) identify pertinent clinical features that may be associated with specific diseases, and 3 ) determine if there are specific patterns of presenting symptoms and other clinical features that could improve the efficiency of the diagnostic process.

\section{Materials and Methods}

A cohort of 184 pediatric patients who received medical care at Winnipeg Children's Hospital between 1991 and 2008 was established retrospectively from several hospital databases and other resources. ${ }^{4}$ All selected children had chronic ataxia and were under the age of 17 years at presentation. Patients included in the study were diagnosed with a wide range of disorders. Ethics approval was granted by the Research Ethics Board of the University of Manitoba.

The inclusion criteria were:

1. Patients were under the age of 17 years on first presentation.

2. Patients had chronic ataxia (i.e. longer than two months or it was recurrent).

3. Patients were assessed and investigated at Winnipeg Children's Hospital.

4. Presentation was during an 18-year period starting in 1991.

The exclusion criteria were:

1. Patients whose ataxia was not a significant clinical feature of their disease.

2. Patients whose ataxia lasted 2 months or less.

3. Patients whose ataxia was caused by primary brain tumor or by disorders solely involving the peripheral nervous system or vestibular system.

4. Patients who were clumsy (i.e. have developmental coordination disorder) but not ataxic.

Further details on methodology have already been published on this cohort with studies focusing on epidemiology, ethnicity, and neuro-ophthalmological features of pediatric patients with chronic ataxia. ${ }^{4,15,16}$ One hundred and sixty nine variables were extracted from the patients' hospital charts including basic demographics, presenting symptoms, other common symptoms, pregnancy history and complications, past medical history, family history, drug history, physical exam findings and investigation results. In this study, data on 151 patients with 16 of the more common diagnoses (defined arbitrarily as present in at least four patients) were then summarized in 65 tables (available on request). The data were checked twice and were subsequently inspected to identify pertinent clinical features among the different diseases that could potentially improve the efficiency of the diagnostic process.

Statistical Package for the Social Sciences (SPSS) version 22 (IBM, Armonk, NY, USA) was used for analysis of the data including calculation of frequencies of the clinical features. Mean, median, standard deviation, range, kurtosis, and skewness for continuous variables were also analyzed. Where data displayed normal distribution, mean and standard deviation were reported. For skewed data, median and range were reported. 
Univariate analysis: Univariate analysis was conducted on the clinical features among nine of the more common diseases (due to statistical limitations of the data) using Pearson Chi-Square test for categorical variables and Kruskal-Wallis test for continuous variables (total number of patients $=109$ ). Results were considered significant when $p<$ 0.05 . The nine diseases were Angelman syndrome $(n=16)$, ataxia telangiectasia $(n=13)$, mitochondrial disease $(n=9)$, Friedreich ataxia $(n=7)$, episodic ataxia $(n=7)$, neuronal ceroid lipofuscinosis $(N C L)(n=6)$, intractable epilepsy syndrome $(n=5)$, neuronal migration disorder (NMD) $(n=5)$, and non-progressive ataxia not yet diagnosed (NYD) $(n=41)$. The purpose of the univariate analysis was to describe the frequency of each of the clinical features among the diseases, and to investigate whether the frequencies differed significantly among them. Due to the large number of diagnoses and rare clinical features, the Pearson chi-square and Kruskal-Wallis tests should be considered preliminary and exploratory.

Latent class analysis (LCA): LCA is a statistical method for detecting multiple sets of patterns among responses to categorical items, questions, or features, which are used to infer the existence of homogeneous subgroups and provide insights into their nature. It attempts to assign mutually exclusive class membership (or subgroups) based on latent patterns among observed variables. ${ }^{17}$ Membership in a class is considered latent or hidden because membership in it cannot be directly observed. This is akin to various clinical features being linked to a diagnosis that is unknown on the initial clinic visits.

The goal of the LCA was to find patterns of clinical features among children with chronic ataxia. Such patterns may be useful to clinicians during the initial clinical assessments, and may offer insights for better understanding the etiology and pathology. We focused on 1) presenting symptoms and 2) other clinical features in this study. Each presenting symptom and clinical feature was considered to be a useful class descriptor if its prevalence differed substantially between classes. From a clinical perspective, it was decided that variables with high probabilities of occurrence needed to be at least $20 \%$ larger when compared to the other classes to be considered as clinically relevant to a particular class in our study. Variables with low probabilities across the classes needed to be approximately 2-3 times larger to be considered as clinically relevant for a particular class. The latent classes were then compared to the diagnoses by using the likeliest predictor class for each subject, based on their responses to the symptom or clinical items as appropriate. The models were performed using PROC LCA, an auxiliary procedure for Statistical Analysis System (SAS), which can be downloaded from Penn State University. ${ }^{17}$ The latent class analyses were conducted on all 184 patients.

LCA of the presenting symptoms: Each of the selected presenting symptoms had to be present in at least 10 patients for it to be included in the LCA. The 14 presenting symptoms that were investigated in the LCA were: Global developmental delay, developmental regression, gross motor delay, speech delay, headache, dizziness, seizures, tremor, head titubation, visual abnormalities, dysarthria, hypotonia, weakness and gait abnormality. All presenting symptoms were dichotomous variables (i.e. present/ absent).

LCA of the other clinical features: Given the constraints of this retrospective study with missing or incomplete data, significant statistical results found in the univariate analysis and clinical knowledge of the diseases investigated were both used to select important clinical features for the LCA. The second LCA was performed on 14 other clinical 
features. The clinical features investigated were: Age of symptom onset, age of ataxia onset, seizure type, sleep difficulty, developmental status, family history of ataxia, consanguinity, dysmorphism, speech difficulty, head titubation, fundus examination, muscle strength, lower limb reflexes, and symptom change over time. Seven of 14 clinical features were dichotomous variables (i.e. absent/ present). Age at symptom onset, age of ataxia onset, seizure type, developmental status, speech abnormalities, lower limb reflexes, and symptom change over time had multiple responses and were converted to categorical variables (i.e. divided into three or more categories).

LCA Models: LCA models with two, three and four class membership were first investigated. Larger models were inestimable given sample size constraints. The LCA models for best fit were chosen based on statistical criteria in combination with clinical knowledge about the diseases investigated in this study. Statistical criteria were based on the lowest Akaike Information Criteria (AIC), Bayesian Information Criteria (BIC), $G^{2}$ values. ${ }^{17}$

Fourteen disease categories were then associated with each class of the chosen models. Each disease had at least five patients as follows: Angelman syndrome $(n=16)$, ataxia telangiectasia $(n=13)$, ischemic stroke $(n=9)$, mitochondrial disease $(n=9)$, Friedreich ataxia $(n=7)$, episodic ataxia $(n=7), N C L(n=6)$, intractable epilepsy syndrome $(n=5)$, hypoxic ischemic encephalopathy after birth (HIE) $(n=5)$, NMD $(n=5)$, Joubert syndrome and related disorders (JS) $(n=5)$, non-progressive ataxia NYD $(n=41)$, and intermittent ataxia NYD $(n=6)$. The uncommon diagnoses (i.e. present in less than five patients) were grouped under "other" $(n=50)$. The total number of patients was 184 .

\section{Results}

The mean age (SD) at the end of the study on the 184 patients $(M=92)$ was $15(7.7)$ years. The median duration [range] of follow-up was 6.4 [0-28.1] years. The etiology of chronic ataxia was known in 128 patients, who collectively had 37 diagnoses.

Univariate analysis: Univariate analysis on 55 variables revealed 33 statistically significant clinical features and one important trend (nystagmus) among 109 patients with nine of the more common diseases (Table 1). Important findings on univariate analysis were: 1) Patients with Angelman syndrome had static symptoms, dysmorphic features, sleep difficulties, needed speech and physiotherapy, and had normal deep tendon reflexes, 2) patients with ataxia telangiectasia had progressive clinical signs, a positive family history of ataxia, needed physiotherapy and speech therapy, had dyskinesia, and heel to shin incoordination, 3) patients with mitochondrial disease had sleep difficulties, used a wheelchair, needed physiotherapy, had generalized seizures, G-tube, and head titubation, 4) patients with Friedreich ataxia had normal development, developed symptoms and ataxia in late childhood, were older at their first clinical assessment, had a positive family history of ataxia, did not need teaching aids, needed physiotherapy, had decreased strength, decreased/ absent deep tendon reflexes, decreased joint position sense, and pes cavus, 5) patients with NCL had excess oral secretions after birth, developmental delay or regression, consanguineous parents, needed physiotherapy, had sleep difficulties, G-tube, head titubation, abnormal pupillary exam, abnormal optic discs, clonus, and brisk deep tendon reflexes, 6) patients with NMD had static symptoms, a positive family history of ataxia, needed physiotherapy, had speech difficulties, dysmorphism, and normal deep tendon reflexes, 7) patients with episodic ataxia were older at the age of symptom onset, older at the age of their first clinical assessment, did not need physiotherapy, and had normal deep tendon reflexes. 
Other important findings: Dyskinesia in ataxia telangiectasia was present in 7 of 10 patients where it was recorded in the chart $(p=0.004)$; however $49 \%$ of the data in the whole cohort were missing including three patients with ataxia telangiectasia. Joint position sense in Friedreich ataxia was decreased or absent in all 7 patients with the disease $(p=0.001)$; however, $69 \%$ of the data in the whole cohort were missing. In addition, nystagmus in episodic ataxia was reported in 5 of 7 patients with the disease with no missing data. However, this did not achieve statistical significance though it was an important clinical trend $(p=0.06)$.

LCA Models: LCA models with two, three, and four class membership were first investigated. For the LCA of the presenting symptoms, the three-class model was chosen because it had a lowest AIC value (Table 2) and was the best clinical fit. For the LCA of other clinical features, the AIC, BIC, and $G^{2}$ values suggested two or four-class models. However, these models were not clinically useful; in the two class model, most of the data clustered around one of the two classes, while in the four class model the probabilities among the classes for several of the clinical features were similar, and did not segregate the classes into more distinct clinical subgroups. Therefore, a three class model was chosen because it was more plausible, useful, relevant, and was the best clinical fit (Table 2). Results of the LCA are shown in tables 3-6. The tables show the probabilities for each presenting symptom and other clinical feature belonging to each class, and their association with 14 disease categories. The variables and associated diagnoses in each class below are listed in order of highest to lowest probabilities.

\section{LCA of the presenting symptoms (Tables 3 and 4)}

Class one - "Gait abnormality subgroup"

Patients in class one commonly had gait abnormality. Seizures, tremor, speech delay gross motor delay, and head titubation were less frequent. The most common diagnoses associated with class one were Friedreich ataxia, ataxia telangiectasia, HIE, mitochondrial disease, intractable epilepsy syndrome, intermittent ataxia NYD, and NCL. Based on our sample, the estimated prevalence of this class in the general chronic ataxia population is $43.1 \%$.

Class two - "Global developmental delay subgroup"

Patients in class two had global developmental delay almost universally. Hypotonia and seizures occurred relatively commonly. The most common diagnoses associated with class two were Angelman syndrome, non-progressive ataxia NYD, JS, and NMD. Based on our sample, the estimated prevalence of this class in the general chronic ataxia population is $38 \%$.

Class three - "Gait abnormality with visual impairments and headache subgroup" Patients in class three had gait abnormality commonly. Visual abnormalities, headache, dizziness, weakness, and dysarthria were less common symptoms. The most common diagnoses associated with class three were episodic ataxia, ischemic stroke, and intermittent ataxia NYD. Based on our sample, the estimated prevalence of this class in the general chronic ataxia population is $18.9 \%$. This class is the least common of the three. 


\section{LCA of other clinical features (Tables 5 and 6)}

For clinical features with more than two responses, e.g. speech abnormalities, lower limb reflexes, and age of ataxia onset, there was variation in the probability of each response within the same class. For example, patients in class one may have both a high probability of having slurred/ scanning speech and a lower probability of having normal speech.

Class one - "Young age of ataxia onset, developmental delay, and dysarthria subgroup" Patients in class one commonly had slurred or scanning speech, global developmental delay, ataxia onset under six years of age, normal lower limb reflexes, and head titubation. Other less common features were decreased lower limb reflexes, sleep difficulties, normal speech, consanguineous parents, and generalized seizures. The most common diagnoses associated with class one were ataxia telangiectasia, intermittent ataxia NYD, ischemic stroke, and non-progressive ataxia NYD. Based on our sample, the estimated prevalence of this class in the general chronic ataxia population is $35.7 \%$.

Class two - "Young age of ataxia onset, developmental delay, and no/ limited speech content"

Patients in class two frequently had speech difficulties (no/ limited speech content), symptom onset under age two years, global developmental delay, ataxia onset under six years of age, and brisk lower limb reflexes. Dysmorphic features, static symptoms, focal seizures, sleep difficulties, normal lower limb reflexes, consanguinity, generalized seizures, head titubation, and developmental regression were other less common features. The most common diagnoses associated with class two were NCL, Angelman syndrome, NMD, intractable epilepsy syndrome, JS, HIE, and mitochondrial disease. Based on our sample, the estimated prevalence of this class in the general chronic ataxia population is $48 \%$.

Class three - "Older age of ataxia onset and normal development subgroup" Patients in class three had no seizures, ataxia onset after age six years, symptom onset after age six years, normal development, slurred or scanning speech, and a family history of ataxia. Symptoms that may improve over time, decreased lower limb reflexes, decreased strength, normal speech, and abnormal fundus examination occurred less commonly. The most common diagnoses associated with class three pattern were episodic ataxia and Friedreich ataxia. Based on our sample, the estimated prevalence of this class in the general chronic ataxia population is $16.3 \%$. This class is the least common of the three.

Appendix 1 ( $p 10$ ) shows a summary of both LCA and their associated diagnoses.

\section{Discussion}

The clinical symptoms and signs of many diseases that cause chronic ataxia in pediatric patients are well established. ${ }^{1,3,6,9,11,13,18}$ However, there is considerable clinical overlap among disorders associated with chronic ataxia with challenges in establishing the diagnosis on the initial clinic visits. ${ }^{3}$ Although neuroimaging is usually helpful in pediatric patients with persisting or recurrent ataxia, ${ }^{9,11}$ the diagnosis is more challenging when neuroimaging is normal or shows non-specific abnormalities. Choosing Wisely Canada is part of an international campaign to reduce unnecessary tests, treatments and procedures on patients. It encourages medical professionals to question the medical 
culture that "more is better". ${ }^{19}$ It aims to reduce the harm, false positives, stress and resources associated with unnecessary test, procedures, and treatments. Our study aims were in accordance with Choosing Wisely Canada principles, namely to identify specific patterns of presenting symptoms and other clinical features that can potentially reduce the number of tests and procedures and improve the efficiency of the diagnostic process in pediatric patients with chronic ataxia.

After inspection of the data, several clinical features appeared to be potentially important discriminators among some of the diseases, which on univariate analysis were found to be statistically significant. Due to statistical constraints of the data, only some of these variables were investigated using LCA. Some of the more discriminating and statistically significant features (i.e. only common in one-two diseases) were: 1) Patients with Angelman syndrome and NMD had dysmorphic features, 2) patients with ataxia telangiectasia had heel to shin incoordination, 3) patients with mitochondrial disease had generalized seizures, 4) patients with Friedreich ataxia developed symptoms later in childhood, were older at their first clinical assessment (i.e. 6-10 years), had normal development, decreased strength, depressed upper and lower limb reflexes, and pes cavus, 5) patients with episodic ataxia developed symptoms later in childhood and were older at their first assessment. Although only a trend, nystagmus was most commonly seen in patients with episodic ataxia, 6) patients with NCL had developmental delay or regression, consanguineous parents, abnormal pupillary exam, abnormal optic discs, brisk upper and lower limb reflexes, and clonus. These clinical features were consistent with the literature reports on the diseases described. ${ }^{20-29}$

To the best of our knowledge, this study is the first to use LCA to investigate the clinical features in pediatric patients with chronic ataxia. Since patients present at different stages of their disease for assessment, the LCA was performed independently on the presenting symptoms and also on other clinical features, because some of the clinical features may not be present on the initial assessment. We identified three distinct latent classes (or subgroups) within the clinical data.

\section{LCA of the presenting symptoms}

Class one "gait abnormality subgroup" describes a group of children who commonly present with abnormalities in gait. Seizures, tremor, and speech delay are less common. Seizures, if present, will help distinguish this class from class three since both classes one and three patients have gait abnormality. Furthermore, class three "gait abnormality with headache and visual disturbances subgroup" is more likely when visual disturbances, headaches, or dizziness occur, in addition to the gait abnormality. In general, class one pattern was more prevalent than class three pattern.

If a patient's presenting symptoms fit class one pattern, genetic investigations can then be initiated. The two most common diagnoses in class one are Friedreich ataxia and ataxia telangiectasia. Both of these disorders have genetic tests available, ${ }^{3}$ but checking alpha fetoprotein level in the blood first is recommended. ${ }^{6}$ If the blood level of alpha fetoprotein is elevated, then genetic testing for ataxia telangiectasia should be pursued first. Other diseases associated with class one include mitochondrial disease and NCL, which can be specifically tested for using biochemical and genetic tests, brain MRI and magnetic resonance spectroscopy, and if necessary muscle biopsy. ${ }^{3,23,24,26,30}$ Patients with HIE have a history of birth asphyxia supported by brain MRI findings of diffuse cerebral injury. ${ }^{8}$ Electroencephalogram is essential in patients with intractable epilepsy. Patients with class three pattern can be investigated for episodic ataxia by specific 
genetic testing (calcium channel mutations). ${ }^{29}$ A patient with an ischemic stroke usually presents acutely to hospital and neuroimaging commonly confirms the diagnosis.

Class two "global developmental delay subgroup" is distinct from classes one and three. Children in this class very frequently have global developmental delay and present with floppiness (hypotonia) and seizures. They benefit from a multidisciplinary team approach to their health needs and usually require support throughout their life. ${ }^{31}$ If a patient fits class two pattern, Angelman syndrome and NMD should be considered. A brain MRI is essential in diagnosing NMD, ${ }^{28,32}$ while genetic testing is available for Angelman syndrome who typically have normal brain MRI. ${ }^{33}$ Joubert syndrome and related disorders have specific brain MRI features (the molar tooth sign) that is diagnostic. ${ }^{34}$

Inquiring about developmental regression is an essential part of the clinical assessment, however in the LCA model, it infrequently featured in all three classes and was not particularly prominent in any one specific class. In addition, it may not be an apparent or obvious feature in the early stages of neurodegenerative disorders.

\section{LCA of other clinical features}

Class one and class two subgroups are similar in that patients in both classes present more commonly at a younger age. Developmental delay and speech difficulties occur almost universally. However, there are important differences between the two classes. Some patients in class one have decreased lower limb reflexes while many patients in class two have brisk lower limb reflexes. Furthermore, the type of speech difficulty observed will help distinguish between the two classes. No or limited speech content is common in class two patients while scanning or slurred speech is common in class one patients. Sleep difficulties and to a lesser extent consanguinity are more common features in patients in class two than class one. If dysmorphic features or developmental regression are observed, then diseases associated with class two should be considered, while head titubation is more commonly seen in diseases associated with class one.

Class three, "Older age of ataxia onset and normal development subgroup", is the most distinct class but is also the least common. Patients in this group are relatively easily differentiated from patients in classes one and two based on their later age of presentation and normal development. Patients in this class do not have seizures and may have a family history of ataxia. Although not observed frequently, the following clinical features are more commonly associated with class three pattern: Abnormal fundus exam and decreased strength, both of which are less common features of Friedreich ataxia. Further tests to confirm specific diagnoses within the classes are available ${ }^{25,29}$ as mentioned above.

The three classes of presenting symptoms and the three classes of other clinical features are each associated with diseases that are segregated unevenly among the classes with some exceptions (Appendix 1). For example, both Friedreich ataxia and ataxia telangiectasia are associated with class one of the presenting symptoms LCA, while Friedreich ataxia is associated with class three in the LCA of other clinical features with ataxia telangiectasia in class one of other clinical features. This will further narrow the diagnostic possibilities. Similarly, when a patient with the highest probability of belonging to class three for both the presenting symptoms and other clinical features, then the most common diagnostic possibilities will be, 1) episodic ataxia, ischemic stroke, and intermittent ataxia NYD, versus 2) episodic ataxia and Friedreich ataxia respectively. Episodic ataxia is common in both classes and should be investigated first. 
Limitations of this retrospective study are misclassification of symptoms or signs, missing data from the hospital charts, or incomplete information recorded. Therefore, not all of the presenting symptoms or other clinical features could be included in the univariate analysis or LCA. Overall sample size constraints further limited the number of variables in the LCA models. In addition, important clinical features and exam findings may not have been present at the time of patient presentation to clinic, and the low number of responses for some variables may have reduced the power of the study to detect important differences in results. Furthermore, it was not feasible for patients with rare diseases (i.e. present in only one or two patients) to be investigated using LCA. Some diseases with chronic ataxia were not represented in our cohort; therefore, the cohort may not be representative of the general population outside our province. Lastly, non-progressive cerebellar ataxia NYD, intermittent ataxia NYD, and other rare disorders that were combined in the analyses did not always segregate clearly into specific classes. This is expected, as they are composed of a heterogeneous group of disorders.

In conclusion, our investigation has shown that LCA results can be helpful in the assessment of children and adolescents with chronic ataxia, where the underlying etiology is usually elusive, especially initially. There was an association among the three subgroups of presenting symptoms and the three subgroups of other clinical features, with several of the more common diseases that cause pediatric chronic ataxia. The LCA subgroups reduced the list of diagnostic possibilities, which can potentially decrease the time to diagnosis, reduce the number of medical investigations and, decrease the financial costs on the health care system. These objectives are consistent with the goals of Choosing Wisely Canada. ${ }^{19}$

A prospective evaluation of our LCA results to confirm and refine our clinical subgroups will be important in future studies. In addition, investigating the contribution of neuroimaging to the diagnosis and prognosis of pediatric patients with chronic ataxia should be undertaken to improve the efficiency of the diagnostic process further. 


\section{Appendix 1 \\ Summary of latent class analyses in association with diagnoses for (A) presenting symptoms and $(B)$ other clinical features (listed from most to least common)}

\begin{tabular}{|c|c|c|c|}
\hline & Class 1 & Class 2 & Class 3 \\
\hline $\begin{array}{l}\text { (A) Presenting } \\
\text { symptoms with } \\
\text { high } \\
\text { probabilities of } \\
\text { belonging to a } \\
\text { specific class }\end{array}$ & $\begin{array}{l}\text { Gait abnormality } \\
\text { Seizures } \\
\text { Tremor } \\
\text { Speech delay } \\
\text { Gross motor delay } \\
\text { Head titubation }\end{array}$ & $\begin{array}{l}\text { Global developmental delay } \\
\text { Hypotonia } \\
\text { Seizures }\end{array}$ & $\begin{array}{l}\text { Gait abnormality } \\
\text { Visual abnormalities } \\
\text { Headache } \\
\text { Dizziness } \\
\text { Weakness } \\
\text { Dysarthria } \\
\text { Gross motor delay }\end{array}$ \\
\hline $\begin{array}{l}\text { Diagnoses that } \\
\text { were highly } \\
\text { associated }\end{array}$ & $\begin{array}{l}\text { Friedreich ataxia } \\
\text { Ataxia telangiectasia } \\
\text { HIE } \\
\text { Mitochondrial disease } \\
\text { Intractable epilepsy syndrome } \\
\text { NCL and Intermittent ataxia NYD }\end{array}$ & $\begin{array}{l}\text { Angelman syndrome } \\
\text { Non-progressive ataxia NYD } \\
\text { JS and NMD }\end{array}$ & $\begin{array}{l}\text { Episodic ataxia } \\
\text { Ischemic stroke } \\
\text { Intermittent ataxia NYD }\end{array}$ \\
\hline
\end{tabular}

\begin{tabular}{|c|c|c|c|}
\hline & Class 1 & Class 2 & Class 3 \\
\hline $\begin{array}{l}\text { (B) Other } \\
\text { clinical } \\
\text { features with } \\
\text { high } \\
\text { probabilities of } \\
\text { belonging to a } \\
\text { specific class }\end{array}$ & $\begin{array}{l}\text { Slurred or scanning speech } \\
\text { Developmental delay } \\
\text { Ataxia onset: } 2-6 \text { years } \\
\text { Normal lower limb reflexes } \\
\text { Symptom onset: } 2-6 \text { years } \\
\text { Head titubation } \\
\text { Ataxia onset < } 2 \text { years } \\
\text { Decreased lower limb reflexes } \\
\text { Sleep difficulties } \\
\text { Normal speech } \\
\text { Consanguinity } \\
\text { Generalized seizures }\end{array}$ & $\begin{array}{l}\text { No or limited speech content } \\
\text { Symptoms onset }<2 \text { years } \\
\text { Developmental delay } \\
\text { Ataxia onset: } 2-6 \text { years } \\
\text { Brisk lower limb reflexes } \\
\text { Dysmorphic features } \\
\text { Static symptoms over time } \\
\text { Focal seizures } \\
\text { Sleep difficulties } \\
\text { Ataxia onset }<2 \text { years } \\
\text { Consanguinity } \\
\text { Generalized seizures } \\
\text { Head titubation } \\
\text { Developmental regression }\end{array}$ & $\begin{array}{l}\text { No seizures } \\
\text { Ataxia onset }>10 \text { years } \\
\text { Symptoms onset }>10 \text { years } \\
\text { Normal development } \\
\text { Slurred or scanning speech } \\
\text { Family history of ataxia } \\
\text { Symptom improvement } \\
\text { Decreased lower limb } \\
\text { reflexes } \\
\text { Symptoms onset: } 6-10 \text { years } \\
\text { Decreased strength } \\
\text { Ataxia onset: } 6-10 \text { years } \\
\text { Normal speech } \\
\text { Abnormal fundus exam }\end{array}$ \\
\hline $\begin{array}{l}\text { Diagnoses that } \\
\text { were highly } \\
\text { associated }\end{array}$ & $\begin{array}{l}\text { Ataxia telangiectasia } \\
\text { Intermittent ataxia NYD } \\
\text { Ischemic stroke } \\
\text { Non-progressive ataxia NYD }\end{array}$ & $\begin{array}{l}\text { NCL } \\
\text { Angelman syndrome } \\
\text { NMD } \\
\text { Intractable epilepsy } \\
\text { syndrome } \\
\text { HIE } \\
\text { JS } \\
\text { Mitochondrial disease } \\
\text { Non-progressive ataxia NYD }\end{array}$ & $\begin{array}{l}\text { Episodic ataxia } \\
\text { Friedreich ataxia }\end{array}$ \\
\hline
\end{tabular}




\section{References}

1. Pandolfo M, Manto M. Cerebellar and afferent ataxias. Continuum (Minneap Minn). 2013; 19(5): 1312-43.

2. Ryan M, Engle E. Acute ataxia in childhood. J Child Neurol. 2003; 18(5): 309-16.

3. Shevell M, Bernard G. The wobbly child: an approach to inherited ataxias. Semin Pediatr Neurol. 2008; 15: 194-208.

4. Salman MS, Lee E, Tjahjadi A, Chodirker BN. The epidemiology of intermittent and chronic ataxia in children in Manitoba, Canada. Dev Med Child Neurol. 2013; 56: 341-7.

5. Lopez-Bastida J, Perestelo-Perez L, Monton-Alvarez F, Serrano-Aguilar P. Social economic costs and health-related quality of life in patients with degenerative cerebellar ataxia in Spain. Mov Disord. 2008; 23: 212-7.

6. Mariotti C, Fancellu R, Di Donato S. An overview of the patient with ataxia. $J$ Neurol. 2005; 252: 511-8.

7. Poretti A, Benson J, Huisman T, Boltshauser E. Acute ataxia in children: approach to clinical presentation and role of additional investigations. Neuropediatrics. 2013; 44: 127-41.

8. Esscher E, Flodmark O, Hagberg G, Hagberg B. Non-progressive ataxia: origins, brain pathology, and impairments in 78 swedish children. Dev Med Child Neurol. 1996; 38: 285-96.

9. Al-Maawali A, Blaser S, Yoon G. Diagnostic approach to childhood-onset cerebellar atrophy: a 10-year retrospective study of 300 patients. J Child Neurol. 2012; 27: 1121-32.

10. Poretti A, Wolf $N$, Boltshauser $E$. Differential diagnosis of cerebellar atrophy in childhood. Eur J Paediatr Neurol. 2008; 12: 155-67.

11. Poretti A, Boltshauser E, Doherty D. Cerebellar Hypoplasia: Differential Diagnosis and Diagnositic Approach. Am J Med Genet. 2014; 166C: 211-26.

12. Di Donato S, Gellera C, Mariotti C. The complex clinical and genetic classification of inherited ataxias. II. Autosomal recessive ataxias. Neurol Sci. 2001; 22: 21928.

13. Palau $F$, Espinos $C$. Autosomal recessive cerebellar ataxias. Orphanet $J$ of Rare Dis. 2006; 1: 47.

14. Regner S, Wilcox N, Friedman L, et al. Friedreich ataxia clinical outcome measures: natural history evaluation in 410 participants. J Child Neurol. 2012; 27(9): 1152-8.

15. Salman MS, Masood S, Azad M, Chodirker BN. Ethnicity and geographic distribution of pediatric chronic ataxia in manitoba. Can J Neurol Sci. 2014; 41: 29-36.

16. Salman MS, Chodirker BN. Neuro-ophthalmological findings in children and adolescents with chronic ataxia. Neuro-Ophthalmology. 2015; 39(3): 125-31.

17. Lanza S, Collins L, Lemmon D, Schafer J. PROC LCA: A SAS procedure for latent class analysis. Struct Equ Modeling. 2007; 14: 671-94.

18. Scaglia F, Towbin J, Craigen W, et al. Clinical spectrum, morbidity and mortality in 113 pediatric patients with mitochondrial disease. Pediatrics. 2004; 114(4): 925-31.

19. Levinson W, Kallewaard M, Bhatia R, Wolfson D, Shortt S, Kerr E. 'Choosing Wisely': a growing international campaign. BMJ Qual Saf. 2015; 24(2): 167-74.

20. Williams C, Driscoll D, Dagli A. Clinical and genetic aspects of Angelman syndrome. Genet Med. 2010; 12(7): 385-95. 
21. Woods C, Taylor A. Ataxia telangiectasia in the british isles: the clinical and laboratory features of 70 affected individuals. Quarterly J of Med. 1992; 82(298): 169-79.

22. Taylor A, Lam Z, Last J, Byrd P. Ataxia telangiectasia: more variation at clinical and cellular levels. Clin Genet. 2015; 87(3): 199-208.

23. Vedanarayanan V. Mitochondrial disorders and ataxia. Semin Pediatr Neurol. 2003; 10(3): 200-9.

24. Parker C, Evans O. Metabolic disorders causing childhood ataxia. Semin Pediatr Neurol. 2003; 10(3): 193-9.

25. Delatycki M, Corben L. Clinical features of Friedreich ataxia. J Child Neurol. 2012; 27: 1133-7.

26. Haltia M. The neuronal ceroid-lipofuscinoses. J Neuropathol Exp Neurol. 2003; 62: 1-13.

27. Poretti A, Boltshauser E, Huisman T. Congenital brain abnormalities: an update on malformations of cortical development and infratentorial malformations. Semin Neurol. 2014; 34(3): 239-48.

28. Brodtkorb E, Nilsen G, Smevik O, Rinck P. Epilepsy and anomalies of neuronal migration: MRI and clinical aspects. Acta Neurol Scand. 1992; 86: 24-32.

29. Jen J, Kim G, Baloh R. Clinical spectrum of episodic ataxia type 2. Neurology. 2004; 62: 17-22.

30. Jadav R, Sinha S, Yasha T, et al. Clinical, electrophysiological, imaging, ultrastructural description in 68 patients with neuronal ceroid lipofuscinoses and its subtypes. Pediatr Neurol. 2014; 50: 85-95.

31. Kripke C. Primary care for adolescents with developmental disabilities. Prim Care Clin Office Pract. 2014; 41: 507-18.

32. Kato M. Genotype-phenotype correlation in neuronal migration disorders and cortical dysplasias. Front Neurosci. 2015; 9: 2-8.

33. Margolis S, Sell G, Zbinden M, Bird L. Angelman syndrome. Neurotherapeutics. 2015; 12: 641-50.

34. Poretti A, Huisman T, Scheer I, Boltshauser E. Joubert syndrome and related disorders: spectrum of neuroimaging findings in 75 patients. AJNR Am J Neuroradiol. 2011; 32(8): 1459-63. 
Tables

Table 1 Clinical features that were statistically *significant in univariate analyses

\begin{tabular}{|c|c|c|}
\hline Diagnoses & No or $<10 \%$ of data missing & $10-30 \%$ of data missing \\
\hline $\begin{array}{l}\text { Ataxia } \\
\text { telangiectasia }\end{array}$ & $\begin{array}{l}\text { Heel to shin incoordination } \\
\text { Dysarthria } \\
\text { Dysmetria } \\
\text { Wide based gait }\end{array}$ & $\begin{array}{l}\text { Scanning/ slurred speech } \\
\text { Family history of ataxia } \\
\text { No seizures } \\
\text { Require physiotherapy } \\
\text { Require speech therapy }\end{array}$ \\
\hline $\begin{array}{l}\text { Friedreich } \\
\text { ataxia }\end{array}$ & $\begin{array}{l}\text { Present with symptoms later } \\
\text { Older age at } 1^{\text {st }} \text { assessment } \\
\text { Older age at ataxia onset } \\
\text { Depressed UL reflexes } \\
\text { Depressed LL reflexes } \\
\text { Pes cavus } \\
\text { Dysarthria } \\
\text { Dysmetria } \\
\text { Dysdiadochokinesia } \\
\text { Intention tremor } \\
\text { Wide based gait }\end{array}$ & $\begin{array}{l}\text { Normal development } \\
\text { Decreased strength } \\
\text { No seizures } \\
\text { Family history of ataxia } \\
\text { Scanning/ slurred speech } \\
\text { Require physiotherapy }\end{array}$ \\
\hline $\begin{array}{l}\text { Neuronal } \\
\text { ceroid } \\
\text { lipofuscinosis }\end{array}$ & $\begin{array}{l}\text { Clonus } \\
\text { Brisk UL reflexes } \\
\text { Brisk LL reflexes } \\
\text { G-tube } \\
\text { Wide based gait } \\
\text { Wheelchair use }\end{array}$ & $\begin{array}{l}\text { Consanguinity } \\
\text { Abnormal pupillary examination } \\
\text { Abnormal optic discs } \\
\text { Developmental delay or } \\
\text { regression } \\
\text { Excess oral secretions after birth } \\
{ }^{\dagger} \text { Speech difficulties } \\
\text { Require physiotherapy } \\
\end{array}$ \\
\hline $\begin{array}{l}\text { Angelman } \\
\text { syndrome }\end{array}$ & $\begin{array}{l}\text { Dysmorphism } \\
\text { Normal UL reflexes } \\
\text { Normal LL reflexes } \\
\text { Wide based gait }\end{array}$ & $\begin{array}{l}\text { Excess oral secretions after birth } \\
\text { Delayed development } \\
\text { †Speech difficulties } \\
\text { Focal seizures } \\
\text { Require speech therapy } \\
\text { Require physiotherapy }\end{array}$ \\
\hline $\begin{array}{l}\text { Mitochondrial } \\
\text { disorders }\end{array}$ & $\begin{array}{l}\text { G-tube } \\
\text { Dysarthria } \\
\text { Wide based gait } \\
\text { Wheelchair use } \\
\end{array}$ & $\begin{array}{l}\text { Generalized seizures } \\
\text { Delayed development } \\
{ }^{\dagger} \text { Speech difficulties } \\
\text { Require physiotherapy } \\
\end{array}$ \\
\hline $\begin{array}{l}\text { Intractable } \\
\text { epilepsy } \\
\text { syndrome }\end{array}$ & $\begin{array}{l}\text { Intention tremor } \\
\text { Brisk LL reflexes } \\
\text { Wide based gait }\end{array}$ & $\begin{array}{l}\text { Delayed development } \\
\text { Focal seizures } \\
{ }^{\dagger} \text { Speech difficulties } \\
\text { Require speech therapy }\end{array}$ \\
\hline $\begin{array}{l}\text { Neuronal } \\
\text { migration } \\
\text { disorder }\end{array}$ & $\begin{array}{l}\text { Dysmorphism } \\
\text { Normal UL reflexes } \\
\text { Normal LL reflexes } \\
\text { Wide based gait }\end{array}$ & $\begin{array}{l}\text { Delayed development } \\
\text { }{ }^{\dagger} \text { Speech difficulties } \\
\text { Focal seizures } \\
\text { Family history of ataxia } \\
\text { Require physiotherapy }\end{array}$ \\
\hline $\begin{array}{l}\text { Non- } \\
\text { progressive } \\
\text { ataxia NYD }\end{array}$ & $\begin{array}{l}\text { Intention tremor } \\
\text { Wide based gait }\end{array}$ & $\begin{array}{l}\text { Delayed development } \\
\text { No seizures } \\
\text { Require physiotherapy } \\
\text { Require speech therapy }\end{array}$ \\
\hline
\end{tabular}




\section{Episodic ataxia Present with symptoms later Do not require physiotherapy Older age at $1^{\text {st }}$ assessment Normal development Older age at ataxia onset No seizures Normal UL reflexes Family history of ataxia Not wide based gait}

${ }^{*} \mathrm{P}$-values were generated with the Pearson chi-square test. The clinical features shown were present in at least $50 \%$ of patients. Total number of patients in all disease groups $=109$. Bolded features were commonly seen in only 1-2 diseases. UL: upper limbs, LL: lower limbs. ${ }^{\dagger}$ Speech difficulty refers to no or limited speech content.

Table 2 Fit statistics for the latent class analysis

\begin{tabular}{llll}
\hline & 2 class model & 3 class model & 4 class model \\
\hline For presenting symptoms: & & & \\
AIC & 311.4 & 307.0 & 307.5 \\
BIC & 404.6 & 448.5 & 497.2 \\
$G^{2}$ & 253.4 & 219.0 & 189.5 \\
\hline For other clinical features: & 1358.6 & 1336.4 & 1309.2 \\
AIC & 1509.7 & 1564.6 & 1614.6 \\
BIC & 1264.6 & 1194.4 & 1119.2. \\
$G^{2}$ &
\end{tabular}

AIC: Akaike information criteria, BIC: Bayesian information criteria

Table 3 Latent class analysis of presenting symptoms and probability (\%) of class membership

\begin{tabular}{llll}
\hline Presenting symptoms & \multicolumn{3}{c}{ Class Membership } \\
& Class 1 (43.1\%) & Class 2 (38.0\%) & Class 3 (18.9\%) \\
\hline Global developmental delay & 14.98 & $\mathbf{9 5 . 7 0}$ & 0.79 \\
Gross motor delay & $\mathbf{1 1 . 6 7}$ & 0.03 & $\mathbf{7 . 8 2}$ \\
Speech delay & $\mathbf{1 4 . 9 3}$ & 0.20 & 0.10 \\
Developmental regression & 7.12 & 8.13 & 4.81 \\
Visual abnormalities & 0.10 & 4.39 & $\mathbf{3 6 . 7 9}$ \\
Head titubation & $\mathbf{7 . 5 2}$ & 0.02 & 0.09 \\
Headache & 0.11 & 0.03 & $\mathbf{2 5 . 4 6}$ \\
Dizziness & 0.03 & 0.02 & $\mathbf{1 9 . 9 4}$ \\
Dysarthria & 5.26 & 0.03 & $\mathbf{1 6 . 6 4}$ \\
Seizures & $\mathbf{2 2 . 4 2}$ & $\mathbf{2 8 . 9 2}$ & 5.84 \\
Tremor & $\mathbf{1 9 . 8 9}$ & 0.30 & 0.11 \\
Hypotonia & 4.67 & $\mathbf{2 9 . 5 5}$ & 4.74 \\
Weakness & 7.37 & 0.14 & $\mathbf{1 7 . 3 6}$ \\
Gait abnormality & $\mathbf{6 0 . 2 7}$ & 38.64 & $\mathbf{6 6 . 6 0}$
\end{tabular}

Bolded numbers indicate the highest probabilities for presenting symptoms and the class they were assigned to. 
Table 4 Latent class analysis of presenting symptoms and probability (\%) of class membership in association with diagnoses

\begin{tabular}{llll}
\hline Diagnosis & \multicolumn{3}{c}{ Class Membership } \\
& Class 1 & Class 2 & Class 3 \\
& $\mathbf{( 4 6 . 2 \% )}$ & $\mathbf{( 3 9 . 7 \% )}$ & $\mathbf{( 1 4 . 1 \% )}$ \\
\hline Ataxia telangiectasia & $\mathbf{8 4 . 6 2}$ & 7.69 & 7.69 \\
Friedreich ataxia & $\mathbf{8 5 . 7 1}$ & 0.00 & 14.29 \\
Ischemic stroke & $\mathbf{4 4 . 4 4}$ & 0.00 & $\mathbf{5 5 . 5 6}$ \\
Neuronal ceroid lipofuscinosis & $\mathbf{5 0 . 0 0}$ & $\mathbf{3 3 . 3 3}$ & 16.67 \\
Mitochondrial disease & $\mathbf{7 7 . 7 8}$ & 11.11 & 11.11 \\
Angelman syndrome & 12.50 & $\mathbf{8 7 . 5 0}$ & 0.00 \\
Episodic ataxia & 28.57 & 0.00 & $\mathbf{7 1 . 4 3}$ \\
Joubert syndrome and related disorders & 20.00 & $\mathbf{6 0 . 0 0}$ & 20.00 \\
Hypoxic ischemia encephalopathy after birth & $\mathbf{8 0 . 0 0}$ & 20.00 & 0.00 \\
Neuronal migration disorder & 40.00 & $\mathbf{6 0 . 0 0}$ & 0.00 \\
Intractable epilepsy syndrome & $\mathbf{6 0 . 0 0}$ & 40.00 & 0.00 \\
Non-progressive ataxia NYD & 31.71 & $\mathbf{6 3 . 4 1}$ & 4.88 \\
Intermittent ataxia NYD & $\mathbf{5 0 . 0 0}$ & 0.00 & $\mathbf{5 0 . 0 0}$ \\
Other & $\mathbf{4 8 . 0 0}$ & $\mathbf{4 0 . 0 0}$ & 12.00 \\
\hline Bolded numbers indicate the highest probabitis
\end{tabular}

Bolded numbers indicate the highest probabilities for associated diagnoses and the class they were assigned to. NYD: not yet diagnosed

Table 5 Latent class analysis of other clinical features and probability (\%) of class membership

\begin{tabular}{llll}
\hline Other clinical features & \multicolumn{3}{c}{ Class Membership } \\
& $\begin{array}{l}\text { Class 1 } \\
\mathbf{( 3 5 . 7 \% )}\end{array}$ & $\begin{array}{l}\text { Class 2 } \\
\mathbf{( 4 8 . 0 \% )}\end{array}$ & $\begin{array}{l}\text { Class } \mathbf{3} \\
\mathbf{( 1 6 . 3 \% )}\end{array}$ \\
\hline Symptom onset: & 59.10 & $\mathbf{8 4 . 8 7}$ & 0.71 \\
Under 2 years of age & $\mathbf{4 0 . 7 9}$ & 15.06 & 0.29 \\
Between 2-6 years of age & 0.04 & 0.02 & $\mathbf{2 9 . 6 9}$ \\
Between 6-10 years of age & 0.07 & 0.05 & $\mathbf{6 9 . 3 0}$ \\
Over the age of 10 years & & & \\
Ataxia onset: & $\mathbf{3 3 . 7 7}$ & $\mathbf{3 1 . 4 7}$ & 0.31 \\
Under 2 years of age & $\mathbf{5 1 . 0 6}$ & $\mathbf{6 0 . 4 9}$ & 0.54 \\
Between 2-6 years of age & 10.36 & 3.63 & $\mathbf{2 6 . 5 0}$ \\
Between 6-10 years of age & 4.82 & 4.42 & $\mathbf{7 2 . 6 4}$ \\
Over the age of 10 years & & & \\
\hline Symptom change over time: & 26.19 & 15.19 & $\mathbf{4 4 . 0 0}$ \\
Improved & 29.15 & $\mathbf{4 5 . 6 2}$ & 22.51 \\
Static & 44.65 & 39.19 & 33.49 \\
Progressive & & & \\
\hline Development: & 18.70 & 0.07 & $\mathbf{6 2 . 9 1}$ \\
Normal & $\mathbf{7 6 . 1 9}$ & $\mathbf{8 3 . 3 3}$ & 36.94 \\
Delayed & 5.12 & $\mathbf{1 6 . 6 0}$ & 0.15 \\
\hline Regressed & 26.62 & 12.96 & $\mathbf{5 2 . 0 1}$ \\
\hline Family history of ataxia & $\mathbf{1 7 . 5 1}$ & $\mathbf{2 5 . 9 1}$ & 6.17 \\
\hline Consanguinity & $\mathbf{2 0 . 4 5}$ & $\mathbf{3 8 . 1 0}$ & 6.33 \\
\hline Sleep difficulties & 23.61 & $\mathbf{4 8 . 7 5}$ & 4.23 \\
\hline Dysmorphic features & & & \\
\hline
\end{tabular}




\begin{tabular}{llll}
\hline Abnormal fundus examination & 4.91 & 11.09 & $\mathbf{2 2 . 9 0}$ \\
\hline Head titubation & $\mathbf{3 7 . 7 1}$ & $\mathbf{2 0 . 5 4}$ & 5.37 \\
\hline Speech: & & & \\
Normal & $\mathbf{1 9 . 5 3}$ & 0.15 & $\mathbf{2 5 . 8 4}$ \\
Scanning or slurred speech & $\mathbf{7 8 . 8 7}$ & 10.34 & $\mathbf{6 0 . 6 5}$ \\
No or limited speech content & 1.60 & $\mathbf{8 9 . 5 1}$ & 13.51 \\
\hline Seizures: & & & \\
None & 72.24 & 36.42 & $\mathbf{9 9 . 4 2}$ \\
Generalized & $\mathbf{1 7 . 4 8}$ & $\mathbf{2 2 . 4 2}$ & 0.25 \\
Focal & 10.28 & $\mathbf{4 1 . 1 6}$ & 0.32 \\
\hline Decrease muscle strength & 15.99 & 10.77 & $\mathbf{2 6 . 7 5}$ \\
\hline Lower limb reflexes: & & & \\
Normal & $\mathbf{4 6 . 7 6}$ & $\mathbf{3 3 . 3 4}$ & 3.71 \\
Decreased & $\mathbf{2 1 . 4 9}$ & 10.14 & $\mathbf{3 6 . 8 1}$ \\
Brisk & 31.75 & $\mathbf{5 6 . 5 3}$ & $\mathbf{2 6 . 1 0}$ \\
\hline
\end{tabular}

Bolded numbers indicate the highest probabilities for other clinical features and the classes they were assigned to.

Table 6 Latent class analysis of other clinical features and probability (\%) of class membership in association with diagnoses

\begin{tabular}{llll}
\hline Diagnosis & \multicolumn{3}{c}{ Class Membership } \\
& $\begin{array}{l}\text { Class 1 } \\
\mathbf{( 3 7 . 5 \% )}\end{array}$ & $\begin{array}{l}\text { Class 2 } \\
\mathbf{( 4 6 . 2 \% )}\end{array}$ & $\begin{array}{l}\text { Class 3 } \\
\mathbf{( 1 6 . 3 \% )}\end{array}$ \\
\hline Ataxia telangiectasia & $\mathbf{8 4 . 6 2}$ & 15.38 & 0.00 \\
Friedreich ataxia & 28.57 & 0.00 & $\mathbf{7 1 . 4 3}$ \\
Ischemic stroke & $\mathbf{6 6 . 6 7}$ & 0.00 & 33.33 \\
Neuronal ceroid lipofuscinosis & 0.00 & $\mathbf{1 0 0 . 0 0}$ & 0.00 \\
Mitochondrial disease & 33.33 & $\mathbf{5 5 . 5 6}$ & 11.11 \\
Angelman syndrome & 0.00 & $\mathbf{1 0 0 . 0 0}$ & 0.00 \\
Episodic ataxia & 14.29 & 0.00 & $\mathbf{8 5 . 7 1}$ \\
Joubert syndrome and related disorders & 40.00 & $\mathbf{6 0 . 0 0}$ & 0.00 \\
Hypoxic ischemia encephalopathy after birth & 40.00 & $\mathbf{6 0 . 0 0}$ & 0.00 \\
Neuronal migration disorder & 20.00 & $\mathbf{8 0 . 0 0}$ & 0.00 \\
Intractable epilepsy syndrome & 20.00 & $\mathbf{8 0 . 0 0}$ & 0.00 \\
Non-progressive ataxia NYD & $\mathbf{4 6 . 3 4}$ & $\mathbf{4 8 . 7 8}$ & 4.88 \\
Intermittent ataxia NYD & $\mathbf{8 3 . 3 3}$ & 0.00 & 16.67 \\
Other & 32.00 & 44.00 & 24.00 \\
\hline Bolded numbers indigate the highest probabiltes for associan
\end{tabular}

Bolded numbers indicate the highest probabilities for associated diagnoses and the classes they were assigned to. NYD: not yet diagnosed 